\title{
Controlled synthesis of transition metal dichalcogenide thin films for electronic applications
}

\author{
Riley Gatensby ${ }^{a, b}$, Niall McEvoy ${ }^{a}$, Kangho Lee ${ }^{a}$, Toby Hallam $^{a}$, Nina C. Berner ${ }^{a}$, \\ Ehsan Rezvani ${ }^{a, b}$, Sinéad Winters ${ }^{a, b}$, Maria O’Brien ${ }^{a, b}$, Georg S. Duesberg ${ }^{a, b, *}$ \\ a Centre for Research on Adaptive Nanostructures and Nanodevices (CRANN), Trinity College Dublin, Dublin 2, Ireland \\ b School of Chemistry, Trinity College Dublin, Dublin 2, Ireland
}

\section{A R T I C L E I N F O}

\section{Article history:}

Received 6 December 2013

Received in revised form 15 January 2014

Accepted 16 January 2014

Available online xxx

\section{Keywords:}

2D materials

Transition metal dichalcogenides

Nanoelectronics

Sensors

Spectroscopy

Thin films

\begin{abstract}
A B S T R A C T
Two dimensional transition metal dichalcogenides (TMDs) are exciting materials for future applications in nanoelectronics, nanophotonics and sensing. In particular, sulfides and selenides of molybdenum (Mo) and tungsten $(\mathrm{W})$ have attracted interest as they possess a band gap, which is important for integration into electronic device structures. However, the low throughput synthesis of high quality TMD thin films has thus far hindered the development of devices, and so a scalable method is required to fully exploit their exceptional properties. Within this work a facile route to the manufacture of devices from $\mathrm{MoS}_{2}$ and $\mathrm{WS}_{2}$, grown by vapour phase sulfurisation of pre-deposited metal layers, is presented. Highly homogenous TMD films are produced over large areas. Fine control over TMD film thickness, down to a few layers, is achieved by modifying the thickness of the pre-deposited metal layer. The films are characterised by Raman spectroscopy, electron microscopy and X-ray photoelectron spectroscopy. The thinnest films exhibit photoluminescence, as predicted for monolayer $\mathrm{MoS}_{2}$ films, due to confinement in two dimensions. By using shadow mask lithography, films with well-defined geometries were produced and subsequently integrated with standard microprocessing process flows and electrically characterised. In this way, $\mathrm{MoS}_{2}$ based sensors were produced, displaying sensitivity to $\mathrm{NH}_{3}$ down to $400 \mathrm{ppb}$. Our device manufacture is versatile, and is adaptable for future nanoscale (opto-) electronic devices as it is reproducible, cost effective and scalable up to wafer scale.
\end{abstract}

(c) 2014 Elsevier B.V. All rights reserved.

\section{Introduction}

The novel properties of two dimensional semiconducting transition metal dichalcogenides (TMDs) make them of great interest for both academia and industry [1-3]. They offer an exciting alternative to graphene, as they possess a band gap, which is crucial for applications in electronics and photonics. These materials have the general formula $\mathrm{MX}_{2}$ with $\mathrm{M}$ being a transition metal (commonly, but not limited to, Mo, W, Nb, Ta, Ti); and X being a chalcogen ( $\mathrm{S}$, Se, $\mathrm{Te}$ ). This class can form two-dimensional layered films composed of a plane of metal atoms covalently bonded to chalcogen atoms. Bulk crystals of such materials have long been studied[4], but in recent years the isolation of their 2D analogues has led to renewed interest in this field.

\footnotetext{
* Corresponding author at: Centre for Research on Adaptive Nanostructures and Nanodevices (CRANN), Trinity College Dublin, Dublin 2, Ireland.

E-mail address: duesberg@tcd.ie (G.S. Duesberg).
}

Importantly, a transition from an indirect gap to a direct gap semiconductor has been reported for $\mathrm{MoS}_{2}$ [5], and $\mathrm{WS}_{2}[6,7]$ as they approach monolayer thickness. Single layer $\mathrm{MoS}_{2}$ shows a transition from the bulk indirect band gap of $1.2 \mathrm{eV}$, to a direct band gap of $1.9 \mathrm{eV}[8]$. The $\mathrm{WS}_{2}$ analogue shows a similar transition with a bulk indirect band gap and monolayer direct band gap of $1.4 \mathrm{eV}$ and $1.8 \mathrm{eV}$, respectively [9]. Recent work has used micromechanically exfoliated layers to produce devices which demonstrate excellent on/off ratios and rapid switching [10-12]. Layers exfoliated by this method have been shown to display photoluminescence (PL) [13] and electroluminescence [14], and thus have potential applicability in optoelectronic devices. However, this production method is very laborious and offers no prospect of scalability.

Scalable production of 2D TMDs has recently been achieved using sonication assisted liquid phase exfoliation in organic solvents [15] and surfactant media [16] as well as by Li intercalation $[17,18]$ and by thermal decomposition of tetrathiomolybdates (or tetrathiotungstates) [19]. Such methods can be used to produce bulk quantities, well suited for applications in composites [20-23], catalysis [19], lithium ion batteries [24,25] and supercapacitors 
[26]. However, they are not readily compatible with standard microprocessing techniques; and the films produced from individual flakes display inferior electronic properties to those obtained by mechanical exfoliation [27]. Chemical vapour deposition (CVD) is a versatile, cost effective and industry compatible technique, which has greatly advanced graphene research in recent years by making high quality films readily available [28,29]. Similar methods can be used for the production of TMD films, and several routes to the production of $\mathrm{MoS}_{2}$ thin films have recently been outlined. These typically are not pure CVD processes but rather involve thermally assisted sulfurisation of Mo [30-32] or, more commonly, $\mathrm{MoO}_{3}$ [33-36], however the use of liquid phase precursors has also been demonstrated $[37,38]$. $\mathrm{WS}_{2}$ has similarly been produced through the thermally assisted sulfurisation of $\mathrm{WO}_{3}$ [33] and $\mathrm{W}$ [39] or by atomic layer deposition [40]. However, fine control of TMD layer thickness over large areas, in combination with structured growth, has seldom been demonstrated.

Within this work a facile route to manufacture of devices from TMDs, grown by vapour phase sulfurisation, is presented. Highly homogenous TMD films are produced over large areas by sulfurisation of pre-deposited metal layers. This is demonstrated for $\mathrm{MoS}_{2}$ and $\mathrm{WS}_{2}$, but can potentially be extended to produce other TMDs. The thickness of the samples is well controlled, from bulk down to monolayer, and high quality homogenous films are produced over a centimetre scale. The uniformity of these films, as observed through scanning Raman analysis, signifies an advance on existing reports on the sulfurisation of Mo which typically show non-uniform films with limited thickness control [31]. By using shadow masks we obtain films with well-defined geometries that can be readily integrated with standard micro-processing technologies. This procedure minimises processing steps, and results in quick, cost effective and versatile device manufacture.

One area of application, which is highly technologically relevant, is chemical sensors. The ever increasing demand for highly sensitive, low cost and low power sensors, in particular for standalone and mobile systems, has necessitated the investigation of new materials. Electronic gas sensors, based on 1D and 2D nanomaterials in a field effect transistor (FET) configuration, have shown considerable promise [41-43]. Improvements in their sensitivity and selectivity have continuously been demonstrated through covalent or non-covalent functionalisation [44-46]. As a semiconducting analogue of graphene, which has demonstrated single molecule detection [43], 2D MoS 2 is an exciting candidate for future sensors. A number of $\mathrm{MoS}_{2}$ based gas sensors have recently been reported using micromechanically exfoliated $[11,47,48]$ and liquid phase exfoliated $[49,50] \mathrm{MoS}_{2}$ flakes. However, these approaches suffer from the previously mentioned problems of poor scalability and poor electronic quality, respectively and it is only recently that devices from more scalable processes have been reported [51]. In this study, we show that our simple process flow is capable of producing gas sensing devices with ultra-high sensitivities, down to 400 ppb for ammonia.

\section{Experimental}

Thin metal films (Mo, W, 99.99\% MaTecK) were sputtered onto substrates ( $300 \mathrm{~nm} \mathrm{SiO}_{2}$ on $\mathrm{Si}$ and fused quartz, Alfa Aesar) using a Gatan Precision Etching Coating System (PECS) allowing for variable thicknesses $(0.5-20 \mathrm{~nm})$. The film deposition rate, and thickness, was monitored using a quartz crystal microbalance, maintaining a deposition rate of $<0.1 \mathrm{~nm} / \mathrm{s}$. Hard masks were used to pattern the coatings, thus defining selective areas of film growth.

Substrates were placed in a quartz tube furnace (Lindberg Blue) and heated $\left(\sim 50^{\circ} \mathrm{C} / \mathrm{min}\right)$ to $500^{\circ} \mathrm{C}$ under Ar flow $(150 \mathrm{sccm}$, $P \sim 0.7$ Torr). After a $5 \mathrm{~min}$ dwell at $500^{\circ} \mathrm{C}$, the samples were heated to $750^{\circ} \mathrm{C}\left(25^{\circ} \mathrm{C} / \mathrm{min}\right)$ and annealed for $30 \mathrm{~min}$. A second upstream hot zone was used to melt S powder (MaTecK, 99\%) to $113 \pm 0.1^{\circ} \mathrm{C}$, and thus introduce $\mathrm{S}$ vapour into the reaction zone. This hot zone consisted of an assembly of halogen bulbs coupled with a power supply. A k-type thermocouple, placed alongside the S supply, allowed for the temperature in the vicinity of the $\mathrm{S}$ powder to be monitored. Following sulfurisation, the samples were held at $750^{\circ} \mathrm{C}$ for a further $20 \mathrm{~min}$, before the furnace was cooled. Upon removal from the furnace, samples were cleaned with acetone and then isopropanol (both HPLC grade) to remove unreacted residue.

For deposition of metal leads a hard mask was aligned to the prestructured TMD films and Ti/Au (10/80 nm) was deposited with the Gatan PECS.

High resolution transmission electron microscopy (HRTEM) bright field images were obtained using a FEI Titan 80-300 TEM. Samples were prepared by first spin coating a PMMA support layer onto the $\mathrm{MoS}_{2}$ thin films and then etching the substrate with $15 \mathrm{M}$ $\mathrm{KOH}$. Samples were then transferred to lacey carbon TEM grids and the PMMA removed by immersion in acetone.

Raman analysis was performed using a Witec Alpha 300R confocal Raman microscope, utilising a $532 \mathrm{~nm}$ laser and an 1800 lines/mm grating. A laser power of less than $1 \mathrm{~mW}$ was used for all measurements in order to avoid sample damage. Raman maps were obtained by taking 4 scans every $\mu \mathrm{m}$ in the $x$ and $y$ direction. PL measurements were taken using the same system with a 600 lines/mm grating in a high $\mathrm{cm}^{-1}$ regime.

X-ray photoelectron spectroscopy (XPS) was performed under ultra-high vacuum conditions (base pressure of $2 \times 10^{-10} \mathrm{mbar}$ ) using a VG Scientific CLAM2 analyser operated at a pass energy of $50 \mathrm{eV}$ with $2 \mathrm{~mm}$ slits, giving a FWHM on $\mathrm{Ag} 3 \mathrm{~d}_{5 / 2}$ of $1.25 \mathrm{eV}$ for the source (PSP twin anode, unmonochromatised Al $\mathrm{K}_{\alpha}$, operated at $12 \mathrm{kV}$ and $12 \mathrm{~mA}$ ). The core-levels were fitted using the software CasaXPS.

Electrical characteristics were measured under ambient conditions using a Karl Suss probe station in conjunction with a Keithley model 2612A source meter.

Gas Sensing measurements were performed in a homemade gas sensing chamber. $200 \mathrm{ppm} \mathrm{NH}_{3}$ gas, balanced by dry $\mathrm{N}_{2}$, was introduced into the chamber using mass flow controllers to dilute the gas concentration by mixing with dry $\mathrm{N}_{2}$. At a $100 \mathrm{sccm}$ flow of the $\mathrm{NH}_{3} / \mathrm{N}_{2}$ mixture, the gas sensing chamber was kept at a pressure of 10 Torr. The electrical resistance of $\mathrm{MoS}_{2}$ sensors was measured using a Keithley model 2612A source meter at a constant bias voltage of $0.2 \mathrm{~V}$ and all measurements were performed at room temperature. In every test, the $\mathrm{MoS}_{2}$ sensors were exposed to pure $\mathrm{N}_{2}$ for 2 min to record their initial resistance and then $\mathrm{NH}_{3}$ gas was introduced for $2 \mathrm{~min}$, and the sensor response measured. The sensors were exposed to $\mathrm{N}_{2}$ for $10 \mathrm{~min}$ to recover and the gas sensing tests were periodically repeated four times.

\section{Results and discussion}

TMD thin films were produced by direct sulfurisation of predeposited metal films in a quartz tube furnace with two heating zones. $\mathrm{MoS}_{2}$ formed the primary focus of our study; however our method was also well-suited to the production of $\mathrm{WS}_{2}$. Thin films of Mo (W) of nominal thicknesses, between 0.5 and $20 \mathrm{~nm}$, were heated to a growth temperature of $750{ }^{\circ} \mathrm{C}$ and $\mathrm{S}$ powder was then melted in a second upstream heating zone. Using Ar as a carrier gas, $\mathrm{S}$ vapour was transported to the substrates, forming $\mathrm{MoS}_{2}$ $\left(\mathrm{WS}_{2}\right)$ at predefined locations. A schematic of this process is shown in Fig. 1a. Films were grown on both $\mathrm{SiO}_{2} / \mathrm{Si}$ and fused quartz substrates by sulfurisation of films with different thicknesses of pre-deposited metal. Photographs of $\mathrm{MoS}_{2}$ films, on both $\mathrm{SiO}_{2} / \mathrm{Si}$ 
(a)

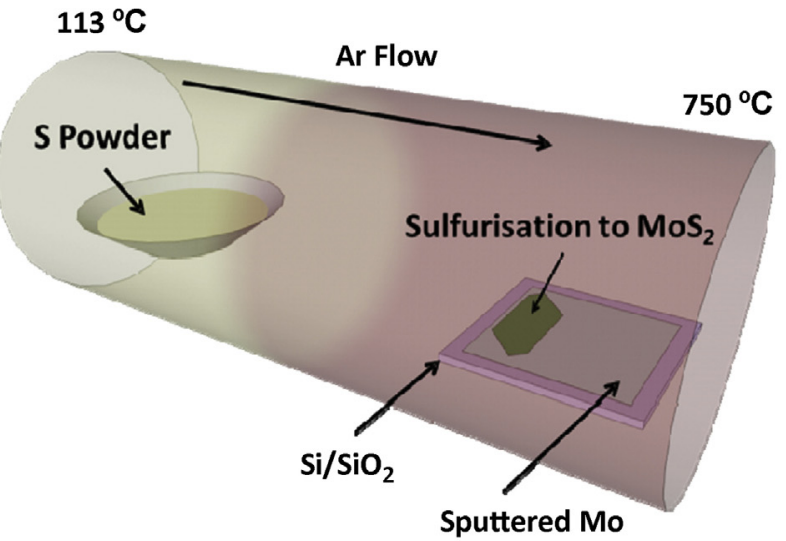

(b)

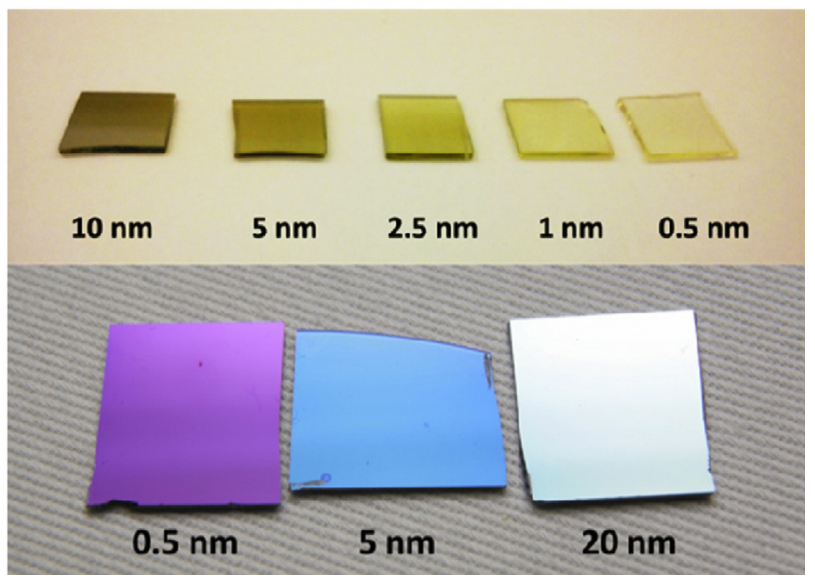

Fig. 1. (a) Schematic diagram of the sulfurisation of Mo. Two distinct heating zones were used; the growth substrates were held at $750^{\circ} \mathrm{C}$, while in a second upstream heating zone S powder was heated to $113^{\circ} \mathrm{C}$. (b) Photograph of $\mathrm{MoS}_{2}$ films formed by sulfurisation of Mo films of different thickness on fused quartz (top) and $\mathrm{SiO}_{2} / \mathrm{Si}$ (bottom) substrates. An increase in opacity with increasing starting film thickness is evident. Each sample shown has an area of $\sim 1 \mathrm{~cm}^{2}$.

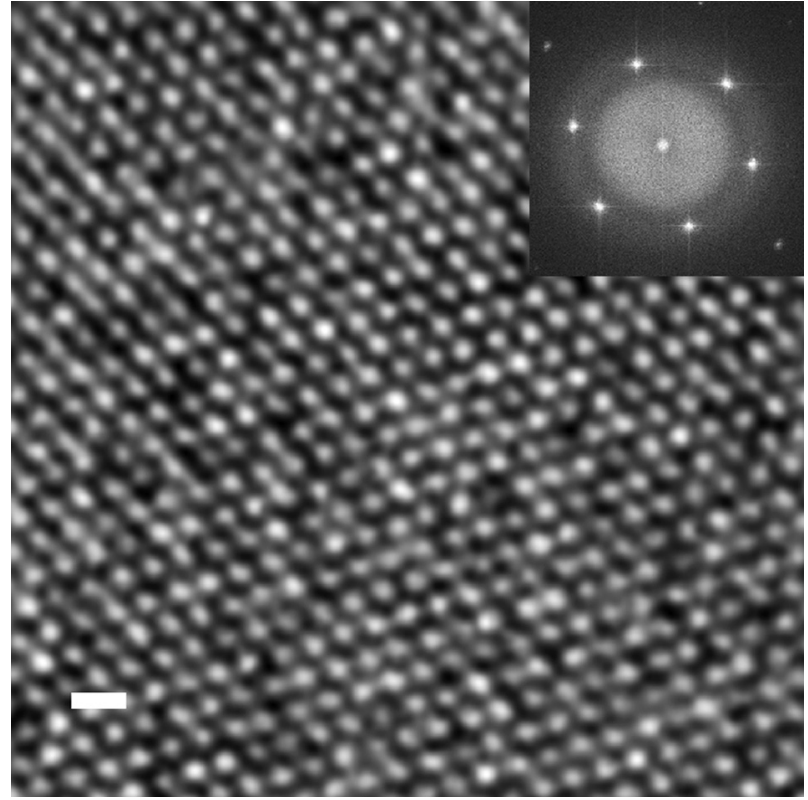

Fig. 2. HRTEM image of a typical $\mathrm{MoS}_{2}$ thin film (nominal Mo thickness of $5 \mathrm{~nm}$ ). Scale bar is $1 \mathrm{~nm}$. Inset is a FFT of the enlarged image showing hexagonal symmetry; sharp spots confirm a very low level of defects.

and fused quartz, are shown in Fig. 1b. Films are easily distinguished from one another, even those with pre-deposited film thicknesses of $0.5 \mathrm{~nm}$ and $1 \mathrm{~nm}$, indicating good control over film thickness. HRTEM was used to probe the crystalline quality of $\mathrm{MoS}_{2}$ films. Films were transferred to grids by etching away the underlying Si substrate in $15 \mathrm{M} \mathrm{KOH}$. An atomic resolution image of a typical thin film ( $5 \mathrm{~nm}$ Mo thickness) is shown in Fig. 2. It clearly shows the hexagonal lattice structure of the film suggesting it is highly crystalline $\mathrm{MoS}_{2}$. The inset shows a fast Fourier transform (FFT) of the HRTEM image. The well-defined spots indicate a low level of (a)

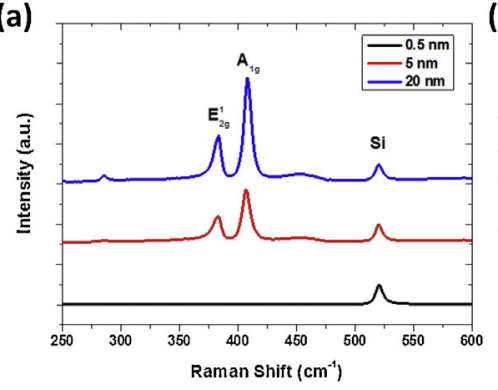

(b)

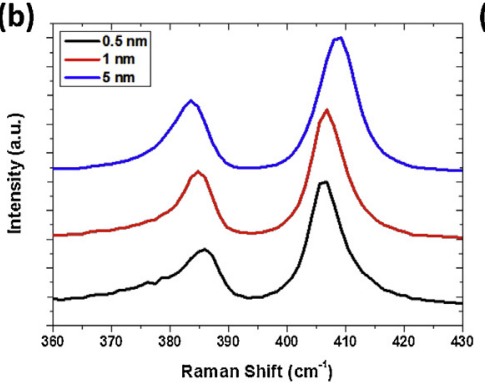

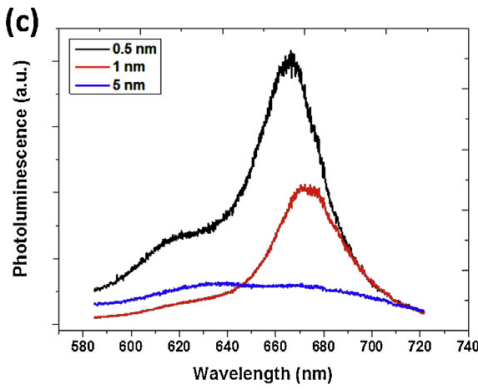

(d)

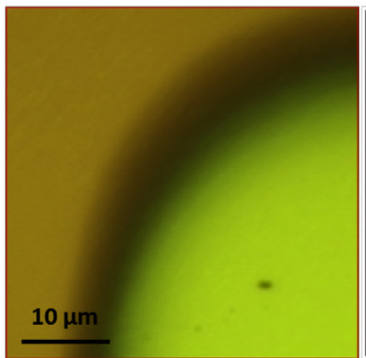

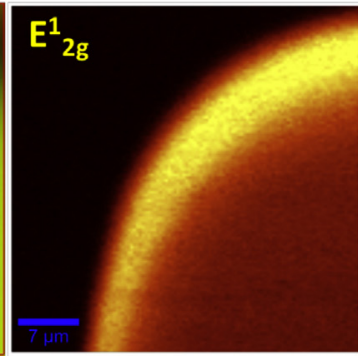
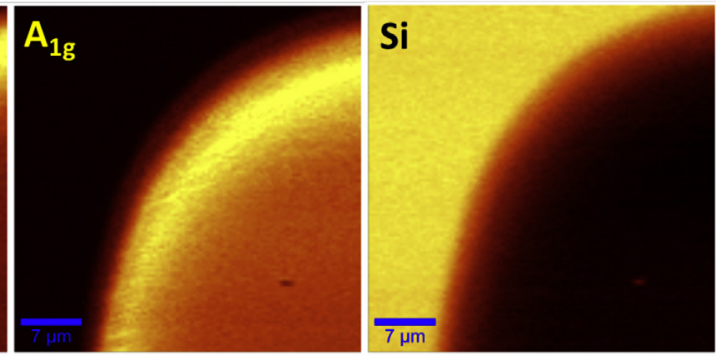

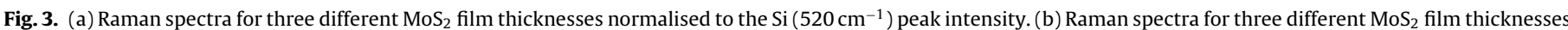

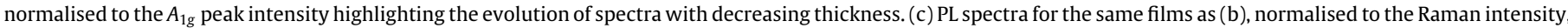

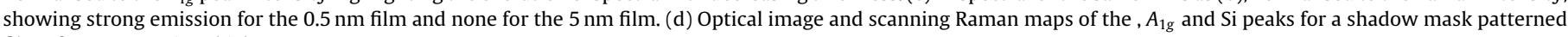
film of $10 \mathrm{~nm}$ starting thickness.

Please cite this article in press as: R. Gatensby, et al., Controlled synthesis of transition metal dichalcogenide thin films for electronic applications, Appl. Surf. Sci. (2014), http://dx.doi.org/10.1016/j.apsusc.2014.01.103 
defects. There was some contamination on the surface of the films, most likely caused by the film transfer process, which involves a PMMA support layer and basic etchant, which could potentially be the cause of the background noise in the FFT. An intensity profile was extracted from the HRTEM image (see supporting information Fig. S1). From this profile, the d-spacing was measured to be $0.63 \mathrm{~nm}$. This is in good agreement with the previously reported spacing of (002) planes of hexagonal $\mathrm{MoS}_{2}$ [52].

Raman spectroscopy was used to assess the layer thickness and uniformity of our films. Bulk $\mathrm{MoS}_{2}$ has two well-known Raman bands at $383 \mathrm{~cm}^{-1}$ and $407.8 \mathrm{~cm}^{-1}$ when probed with a $532 \mathrm{~nm}$ excitation laser [53]. These correspond to $E_{2 g}^{1}$ and $A_{1 g}$ vibrational modes, respectively. Spectra of three films, with different starting Mo thicknesses, grown on $\mathrm{Si} / \mathrm{SiO}_{2}$, are shown in Fig. 3a. It is clear that the relative intensity of the $\mathrm{MoS}_{2}$ bands to the Si peak of the substrate at $520 \mathrm{~cm}^{-1}$ scales with increasing film thickness,
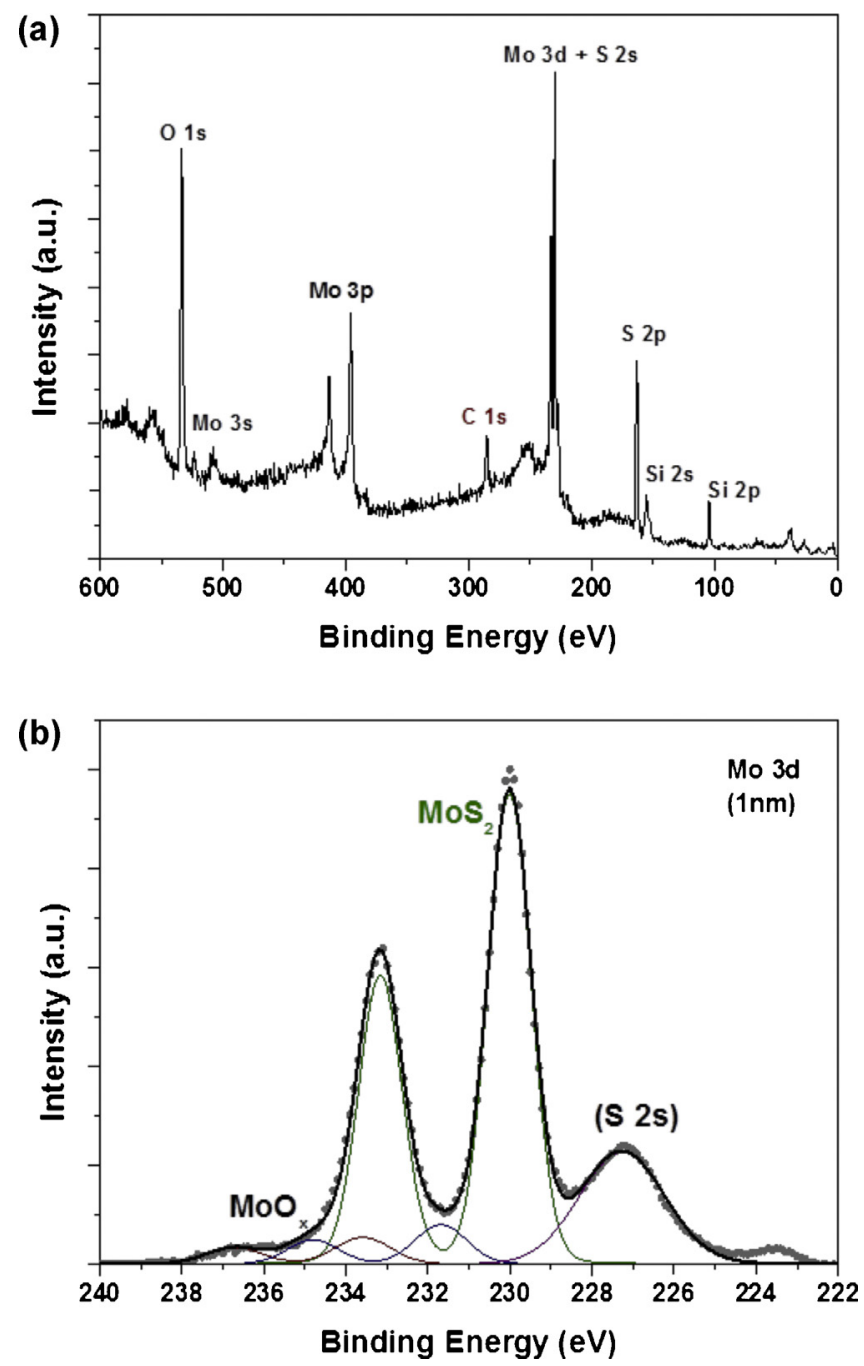

Fig. 4. (a) An XPS survey spectrum for a film with an initial Mo thickness of $1 \mathrm{~nm}$. The difference in binding energy between the Mo $3 d_{5 / 2}$ and the $S 2 p_{3 / 2}$ peaks of $\Delta E=67.1 \mathrm{eV}$ indicates a stoichiometric ratio in our $\mathrm{MoS}_{x}$ samples of $x=1.9 \pm 0.1$. The lack of major contaminants, except natural organic matter (C), is also evident. (b) The Mo 3d core-level with fitted components after subtraction of a Shirley background, also including the S 2s core-level in the same binding energy region. The main component at $230 \mathrm{eV}$ corresponds to sulfurised Mo, with two minor components at $231.7 \mathrm{eV}$ and $233.6 \mathrm{eV}$ originating from residual oxides. The spin-orbit splitting in the Mo $3 \mathrm{~d}$ doublets is $3.25 \mathrm{eV}$ and the branching ratio (Mo $3 \mathrm{~d}_{3 / 2}$ to Mo $3 d_{5 / 2}$ ) is 2:3. All components of the Mo $3 d$ line have been fitted using a mixed Gaussian-Lorentzian line shape with a FWHM of $1.3 \pm 0.1 \mathrm{eV}$. confirming that the thickness of $\mathrm{MoS}_{2}$ produced is directly related to the starting Mo thickness.

Li et al. described the evolution of the Raman signal of mechanically exfoliated $\mathrm{MoS}_{2}$ flakes upon reducing the number of layers. They observed an upward shift of $1.7 \mathrm{~cm}^{-1}$ and a downward shift of $5.1 \mathrm{~cm}^{-1}$ in the and $A_{1 \mathrm{~g}}$ peaks, respectively, upon going from bulk crystals to monolayers[53]. Such a trend was previously predicted from $a b$ initio calculations by Molina-Sánchez et al. [54]. Spectra of three films of different thickness, normalised to the $A_{1}$ geak intensity are shown in Fig. $3 \mathrm{~b}$. As expected, the $E_{2 \mathrm{~g}}^{1}$ and $A_{1 \mathrm{~g}}$ peaks shift closer to one another with decreasing film thickness. In the case of the $5 \mathrm{~nm}$ sample the and $A_{1 \mathrm{~g}}$ peaks are seen at $383.6 \mathrm{~cm}^{-1}$ and $408.6 \mathrm{~cm}^{-1}$, a separation of $25 \mathrm{~cm}^{-1}$, whereas for the $0.5 \mathrm{~nm}$ sample the same peaks manifest at $385.9 \mathrm{~cm}^{-1}$ and $406.3 \mathrm{~cm}^{-1}$, a separation of $20.4 \mathrm{~cm}^{-1}$. This suggests that the $0.5 \mathrm{~nm}$ sample consists of very few (1-3) layers, whereas the $5 \mathrm{~nm}$ sample behaves like bulk $\mathrm{MoS}_{2}$. It is probable that the laser, with a spot size of $\sim 300 \mathrm{~nm}$, probes a number of crystal sites and thus the observed Raman
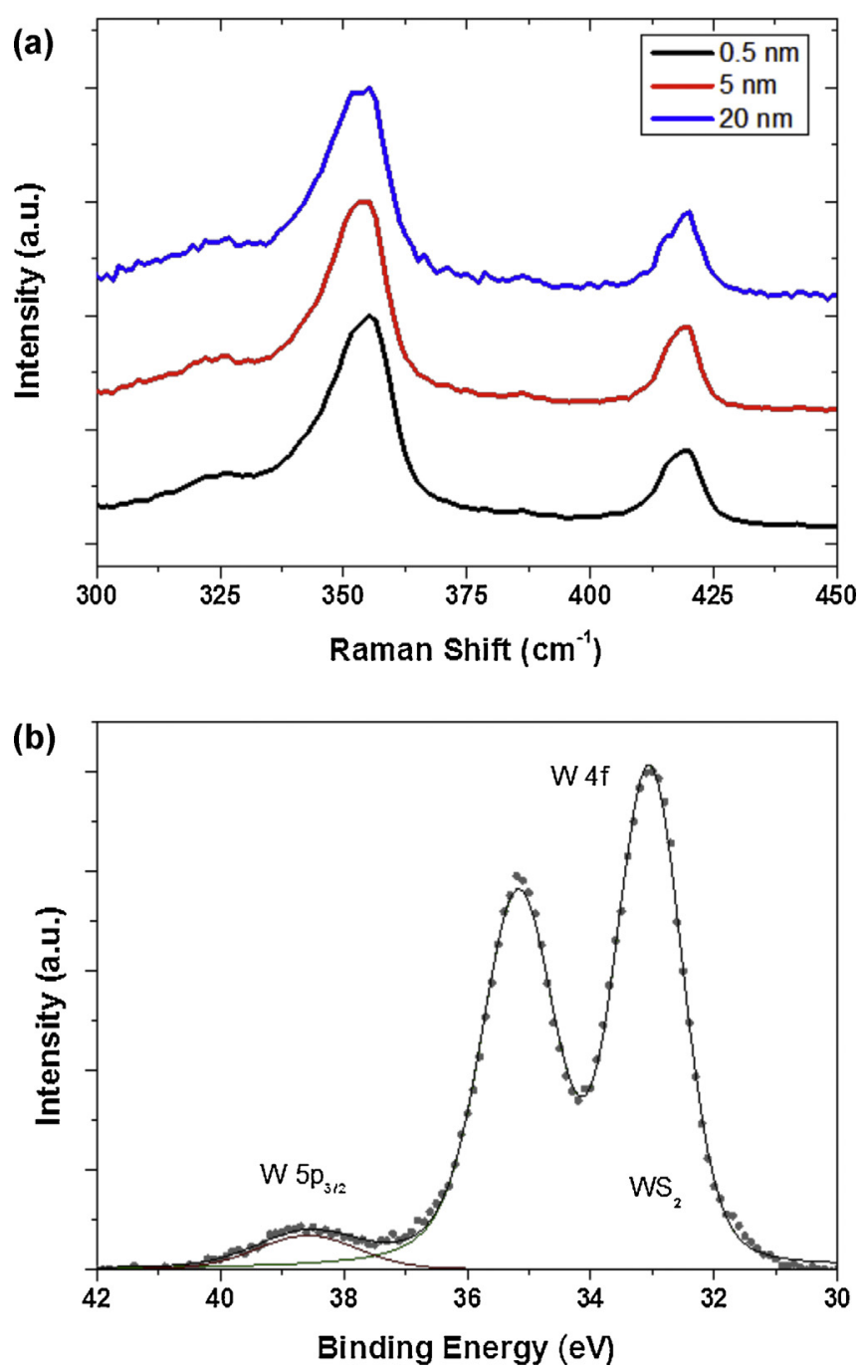

Fig. 5. (a) Raman spectra for three different $\mathrm{WS}_{2}$ film thicknesses normalised to the $A_{1 \mathrm{~g}}$ peak intensity. Unlike the corresponding $\mathrm{MoS}_{2}$ spectra, thickness dependent peak shifts are not immediately obvious. (b) XPS of the W $4 \mathrm{f}$ core-level of a $\mathrm{WS}_{2}$ film with an initial $\mathrm{W}$ thickness of $20 \mathrm{~nm}$, after subtraction of a Shirley background and with the $\mathrm{W} 5 \mathrm{p}_{3 / 2}$ line in the same binding energy range. The $4 \mathrm{f}$ line was fitted with a single component at a binding energy of $33.05 \mathrm{eV}$ (for the $4 \mathrm{f}_{7 / 2}$ ), which indicates completely sulfurised $\mathrm{W}$ at the surface. The spin-orbit splitting in the $\mathrm{W} 3 \mathrm{f}$ doublet is $2.13 \mathrm{eV}$ and the branching ratio $\left(\mathrm{W}_{4} \mathrm{f}_{5 / 2}\right.$ to $\left.\mathrm{Mo} 4 \mathrm{f}_{7 / 2}\right)$ is $3: 4$. The line has been fitted using a mixed Gaussian-Lorentzian line shape with a FWHM of $1.15 \pm 0.10 \mathrm{eV}$. 


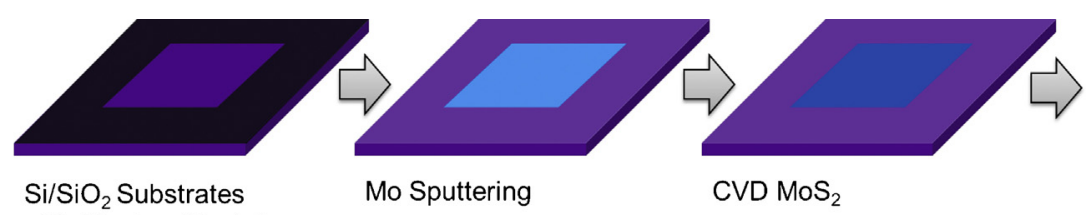
with Shadow Mask 1
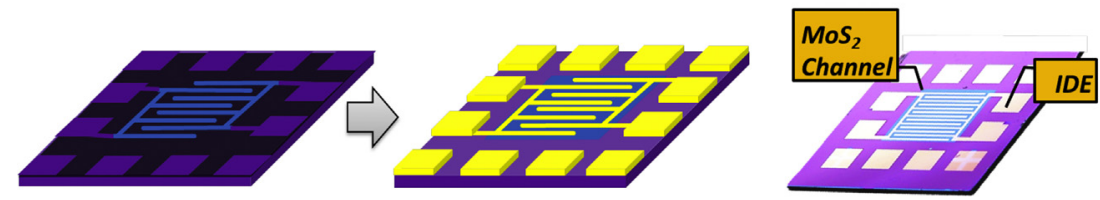

Metal Deposition with Shadow Mask 2

IDE Device

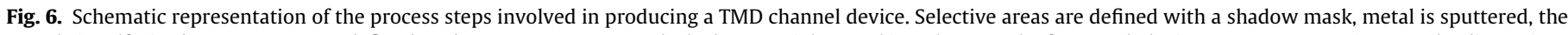

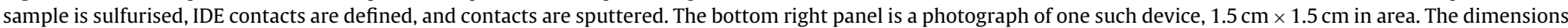

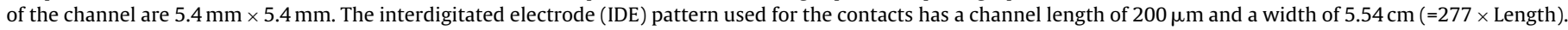

signal may consist of contributions from domains of different layer thickness.

PL studies offer an alternative route to probing the thickness, and electronic properties, of $\mathrm{MoS}_{2}$ films. Splendiani et al. measured PL spectra for $\mathrm{MoS}_{2}$ flakes of different thickness [5]. In bulk $\mathrm{MoS}_{2}$, with its indirect band gap, they observed no PL emission. However, in few- and monolayer crystals they noted PL emissions at the A1 and B1 direct excitonic transitions. PL spectra, as shown in Fig. 3c, were acquired on the same films used for Raman analysis. The PL intensity is normalised to the $\mathrm{MoS}_{2}$ Raman signal in each case. The $5 \mathrm{~nm}$ film shows no PL, further implying that it constitutes bulk material. However, both the $0.5 \mathrm{~nm}$ and $1 \mathrm{~nm}$ films display the PL emissions characteristic of few layer crystals. These spectra were all acquired under identical acquisition conditions and so the high spectral intensity and, relative high intensity of the A1 to B1 emission observed for the $0.5 \mathrm{~nm}$ film imply that it is, at least partially, monolayer in nature. Six separate films with a starting thickness of $0.5 \mathrm{~nm}$ were fabricated and each one showed similar PL behaviour, indicating good reproducibility.

Scanning Raman spectroscopy was employed to assess the uniformity of a $\mathrm{MoS}_{2}$ film over a large area. An optical image and Raman maps of a shadow mask patterned sample, with a starting Mo thickness of $10 \mathrm{~nm}$, are shown in Fig. 3d. These demonstrate good uniformity in the film, with no cracks or tears observed over the scan area. Additionally, no $\mathrm{MoS}_{2}$ is seen outside of the patterned region, signifying good control over feature placement when using shadow masks. Additional Raman maps, highlighting the increase in and $A_{1 g}$ peak separation with increasing thickness are shown in
Fig. S2. Scanning Raman spectroscopy also allowed for PL spectra to be acquired over a large area. A PL surface map of a $\mathrm{MoS}_{2}$ film, with a starting Mo thickness of $0.5 \mathrm{~nm}$, is shown in Fig. S3. This shows a reasonably consistent signal, over a large area, further implying film uniformity, even in the case of very thin films. To the best of our knowledge, such uniform PL has not previously been reported for $\mathrm{MoS}_{2}$ films produced by sulfurisation of Mo.

XPS measurements provided further information about the composition, purity and thickness of our films. They were performed on films which were produced from nominal Mo thicknesses of $0.5,1$ and $5 \mathrm{~nm}$, sputtered onto $\mathrm{Si} / \mathrm{SiO}_{2}$ substrates, as well as on a clean $\mathrm{Si} / \mathrm{SiO}_{2}$ surface as a reference for film thickness estimation. An XPS survey spectrum of a $\mathrm{MoS}_{2}$ film with a starting Mo thickness of $1 \mathrm{~nm}$ is shown in Fig. 4a. Aside from a weak carbon signal, which is generally unavoidable, no other contaminants were observed, indicating the high purity of our samples. In a previous XPS study on bulk $\mathrm{MoS}_{2}$, Baker et al. show that the difference in binding energy between the Mo $3 d_{5 / 2}$ and the $S 2 p_{3 / 2}$ peaks can be related to the stoichiometric composition of the $\mathrm{MoS}_{2}$ [55]. All measured samples showed a $\Delta E$ value of $67.1 \mathrm{eV}$, corresponding to a stoichiometric ratio of $x=1.9 \pm 0.1$. This is closer to the nominal value of 2 for $\mathrm{MoS}_{2}$ than values reported previously for films produced by sulfurisation of $\mathrm{MoS}_{2}$ (2.2) [31]. Furthermore, as shown in Fig. 4b, the Mo 3d core-level can be fitted with one main component at a binding energy of $230 \mathrm{eV}$ for the Mo $3 \mathrm{~d}_{5 / 2}$ peak, which originates from sulfurised Mo, and two smaller components on the high binding energy side of the peak which are most likely related to residual oxides. The $S 2$ p core-level (not shown)
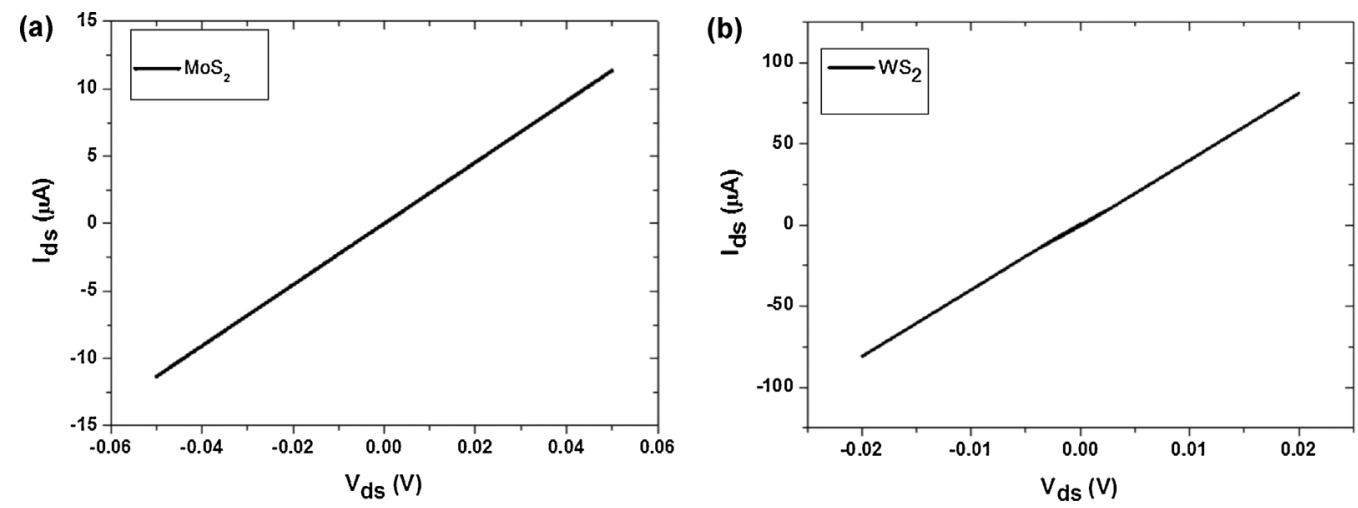

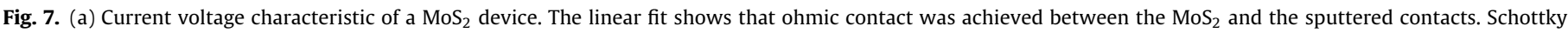

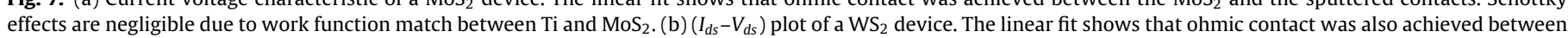
the $\mathrm{WS}_{2}$ and the sputtered contacts. 
could be fitted with only one component at a binding energy of $162.9 \mathrm{eV}$, indicating that there is no unreacted sulfur on the surface.

By comparing the areas of the silicon XPS peaks before and after growth of $\mathrm{MoS}_{2}$ films on the $\mathrm{Si} / \mathrm{SiO}_{2}$ substrates, and using the exponential decay relation of photoelectrons emitted through an overlayer, the film thicknesses of different samples were estimated. The average thickness of a film with an initial nominal Mo thickness of $0.5 \mathrm{~nm}$ was found to be $2.0 \mathrm{~nm}$. This corresponds to $\sim 2-3$ layers, given a typical $\mathrm{MoS}_{2}$ interlayer thickness of $0.66 \mathrm{~nm}$ [31]. Similarly, films with starting thicknesses of $1 \mathrm{~nm}$ and $5 \mathrm{~nm}$ were measured to be $3.9 \mathrm{~nm}$ (6-7 layers) and $6.1 \mathrm{~nm}$ (9-10 layers) following sulfurisation. This is an expected result, as the film will expand upon the addition of $S$ between the Mo layers. These estimated values are in close agreement with the Raman and PL observation which suggest thickness close to a monolayer. This expansion was also observed by atomic force microscopy, as shown in Fig. S4.

$\mathrm{WS}_{2}$ films were also produced and characterised, demonstrating the flexibility of our production method. Raman spectra of such films are similar to those of $\mathrm{MoS}_{2}$, with peaks corresponding to and $A_{1 g}$ vibrational modes. These are observed at $354 \mathrm{~cm}^{-1}$ and $420 \mathrm{~cm}^{-1}$, respectively, as shown in Fig. $5 \mathrm{a}$, which is in good agreement with literature values [56]. XPS analysis of the W $4 \mathrm{f}$ core-level, as shown in Fig. 5b, shows only one component at a binding energy

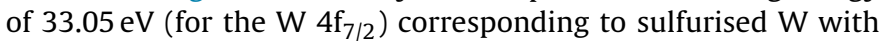
no indication of oxides being present on the surface. Similarly to the previously discussed $\mathrm{MoS}_{2}$ samples, the survey scan shows no major contaminants and the S 2 p core-level could only be fitted with one component at $162.5 \mathrm{eV}$ (both not shown).

Devices were fabricated using selective area deposition, employing a shadow mask during the metal deposition, to create TMD patterns. A second shadow mask was used to pattern metal contacts on the thus derived films. A schematic of this is shown in Fig. 6. The bottom right panel in Fig. 6 is a photograph of such a device. Contacts were formed by sputtering Ti/Au $(10 / 80 \mathrm{~nm})$. The Ti serves two purposes, it acts as an adhesion layer for the gold, and it more closely matches the work function of the $\mathrm{MoS}_{2}$, thereby limiting the height of the contact resistance at the interface. An interdigitated electrode (IDE) pattern, with a channel length of $200 \mu \mathrm{m}$ and a width of $5.54 \mathrm{~cm}$ (=277 $\times$ Length) was used. Additional electrical measurements are included in the supporting information Figs. S6 and S7.

The source-drain current versus source-drain voltage $\left(I_{d s}-V_{d s}\right)$ characteristic, for a pre-deposited Mo thickness of $20 \mathrm{~nm}$, is shown in Fig. 7a. The $20 \mathrm{~nm} \mathrm{MoS}$ film is linear at a bias voltage sweep in the range of $\pm 50 \mathrm{mV}$. The observed behaviour indicates that our device has intimate contact electrodes, with no contribution from a Schottky contact at the interface, thus excluding the possibility of a Schottky barrier, which would suppress charge carrier flow in the semiconducting channel. $\mathrm{WS}_{2}$ devices exhibit the same ohmic $\left(I_{d s}-V_{d s}\right)$ characteristic, as evidenced by the linear nature of Fig. $7 \mathrm{~b}$. The devices exhibit minimal contact resistance.

Fig. 8a shows typical gas sensor response curves of such a device at various concentrations of $\mathrm{NH}_{3}$, from $400 \mathrm{ppb}$ to $200 \mathrm{ppm}$. $\mathrm{NH}_{3}$ is an electron-donating moiety and our sensor devices show n-type behaviour, thus adding majority carriers has the effect of increasing conductivity. A recent study on the gas sensing properties of mechanically exfoliated $\mathrm{MoS}_{2}$ flakes illustrated a selective response to electron donors [47]. When the $\mathrm{MoS}_{2}$ film is exposed to gaseous $\mathrm{NH}_{3}$, adsorbed molecules on the surface of $\mathrm{MoS}_{2}$ shift the Fermi level to the conduction band, resulting in the observed resistance decrease. Although the sensor response curves immediately indicate $\mathrm{NH}_{3}$ injections, even at sub-ppm levels (Fig. 8b), recovery is slow in pure $\mathrm{N}_{2}$ flow at room temperature; and baselines gradually shift downward for consecutive $\mathrm{NH}_{3}$ injections. The surface may exhibit tight bonding with $\mathrm{NH}_{3}$ molecules, as has commonly been (a)
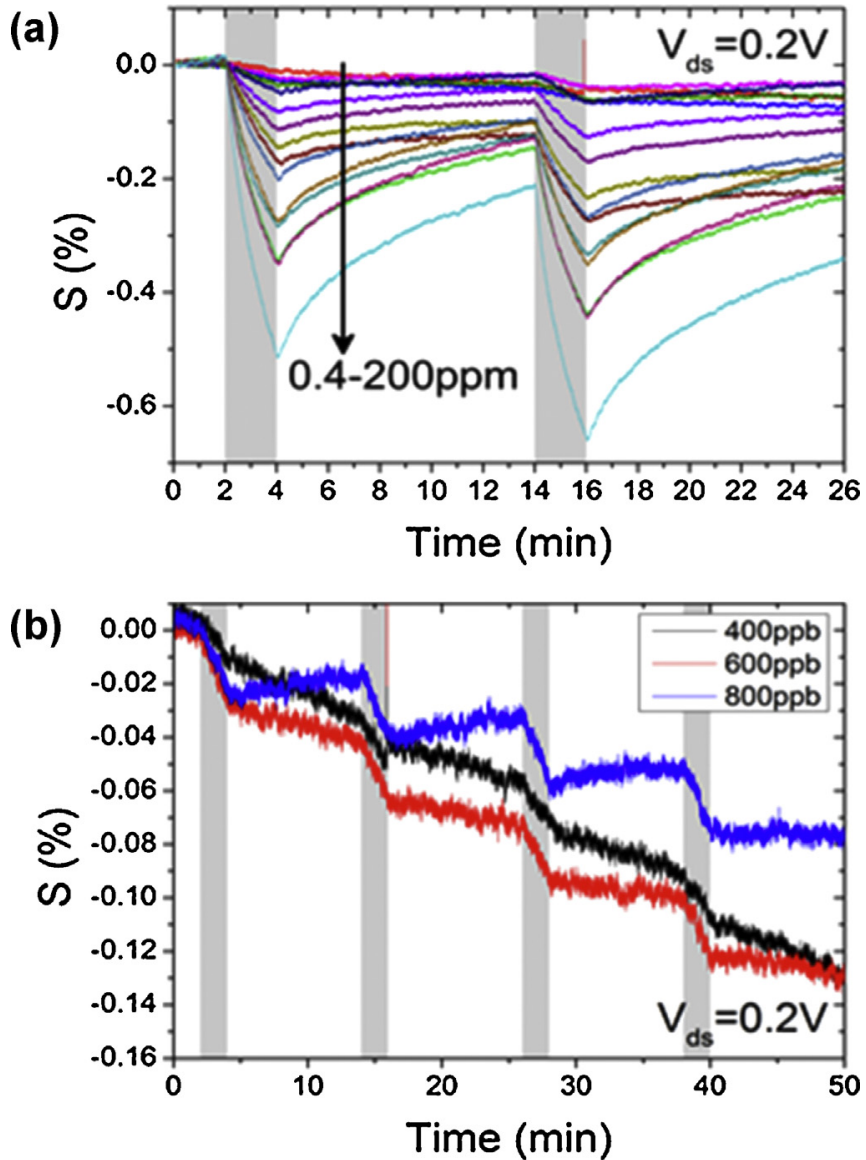

(c)

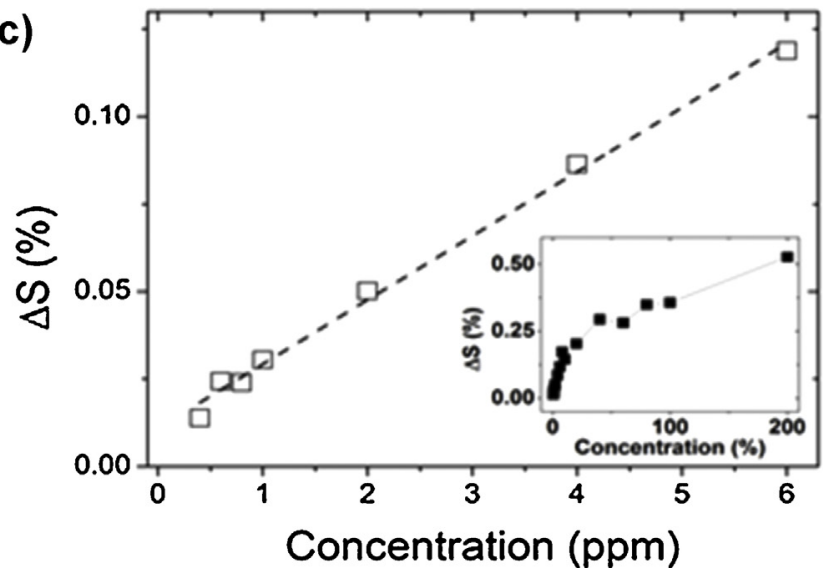

Fig. 8. (a) Sensor response $(S)$ curves of the $\mathrm{MoS}_{2}$ film at various $\mathrm{NH}_{3}$ concentrations from $400 \mathrm{ppb}$ to $200 \mathrm{ppm}$ and (b) four-fold plots of sensor response at 400,600 , and $800 \mathrm{ppb}$. Grey vertical bars indicate $\mathrm{NH}_{3}$ gas injections for $2 \mathrm{~min}$. (c) Plots of sensitivity indicating percentile resistance changes between initial resistance and peak value of the first gas sensing cycle. Open boxes and broken line indicate measured data and fitted line, respectively. Inset: A full-scale sensitivity plot from $400 \mathrm{ppb}$ to 200 ppm.

observed for nanomaterial-based sensors with extremely high sensitivities. The recovery speed could potentially be accelerated by ultra-violet illumination or annealing [43,57].

Sensor responses for ammonia gas injections, over 2 min intervals, define the sensor sensitivity in this study, as shown in Fig. 8c. In this study, sensor response $(S)$ is defined by percentile resistance change, given by the following relation;

$S=\frac{\Delta R}{R_{0}}=\frac{R_{S}-R_{0}}{R_{0}} \times 100 \%$ 
where $R_{0}$ is the initial resistance of the sensor and $R_{S}$ is the resistance at gas introduction. The introduction of ammonia causes a decrease in $S$ because $R_{S}<R_{0}$; therefore $S$ is a negative quantity. The sensor sensitivity is linearly proportional to the concentration of $\mathrm{NH}_{3}$ introduced in the low concentration range, which makes determination of gas concentration feasible, and a full-scale plot shows a hyperbolic curve (inset Fig. 8c). As a result, we obtained a practical detection limit of $400 \mathrm{ppb}$, which could be proven by signal-to-noise ratios (SNRs) based on simple signal processing. From the first 4000 data points (about $2 \mathrm{~min}$ ), the initial resistance and root-mean-square (rms) noise of sensors were derived using the following equation;

$\mathrm{rms}_{\text {Noise }}=\sqrt{\frac{\sum\left(R-R_{0}\right)}{N}}$

where $R$ is the measured resistance and $N$ is the number of data points. According to the IUPAC definition [58], the signal should be at least three times larger than the rms noise. Our $\mathrm{MoS}_{2}$ sensors have a SNR of 8.3 at $400 \mathrm{ppb}$, and the SNR increases with increasing $\mathrm{NH}_{3}$ concentration. Overall, this sensor performance is superior to previous reports on $\mathrm{MoS}_{2}$ based ammonia sensors, produced from liquid phase exfoliated flakes, which show poor recovery at higher concentrations [50]. This low detection limit signifies that our $\mathrm{MoS}_{2}$ sensor is a strong candidate for gas sensing applications. Functionalisation of the channel could engender selectivity in our device, as has been observed previously for other nanomaterial based sensors [44-46]. Sensors such as this, whereby a particular analyte (e.g. $\mathrm{H}_{2} \mathrm{~S}, \mathrm{CO}_{2}$ ) is targeted, are of high commercial interest.

\section{Conclusion}

We have shown a versatile method for the production of devices from TMD films, in particular with $\mathrm{MoS}_{2}$ and $\mathrm{WS}_{2}$. Control over film thickness was attained by modifying the thickness of pre-deposited metal layers. HRTEM studies outlined the crystalline quality of our samples with an FFT representative of hexagonal symmetry. XPS analysis further emphasised the high quality of the films, with minimal impurities present. The thinnest $\mathrm{MoS}_{2}$ films $(0.5 \mathrm{~nm})$ exhibited a photoluminescence signal and Raman spectra, consistent with few-layer material. Films with starting thicknesses of $5 \mathrm{~nm}$ and greater displayed bulk like properties. These films were readily integrated into devices using a facile shadow masking procedure. Gas sensor devices produced in this manner display excellent sensitivity, with a detection limit of $400 \mathrm{ppb}$ for ammonia. The ease of device manufacture, cost effectiveness, scalability and compatibility with existing semiconductor fabrication methods make this process favourable for future sensors and other electronic applications.

\section{Acknowledgements}

The authors thank Science Foundation Ireland (SFI) for financial support under Contract Nos. 08/CE/I1432 and PI_10/IN.1/I3030.

\section{Appendix A. Supplementary data}

Supplementary data associated with this article can be found, in the online version, at http://dx.doi.org/10.1016/j.apsusc. 2014.01.103.

\section{References}

[1] Q.H. Wang, K. Kalantar-Zadeh, A. Kis, J.N. Coleman, M.S. Strano, Electronics and optoelectronics of two-dimensional transition metal dichalcogenides, Nat. Nanotechnol. 7 (2012) 699-712.
[2] M. Chhowalla, H.S. Shin, G. Eda, L.J. Li, K.P. Loh, H. Zhang, The chemistry of twodimensional layered transition metal dichalcogenide nanosheets, Nat. Chem. 5 (2013) 263-275.

[3] X. Huang, Z. Zeng, H. Zhang, Metal dichalcogenide nanosheets: preparation, properties and applications, Chem. Soc. Rev. 42 (2013) 1934-1946.

[4] J.A. Wilson, A.D. Yoffe, The transition metal dichalcogenides discussion and interpretation of the observed optical, electrical and structural properties, Adv. Phys. 18 (1969) 193-335

[5] A. Splendiani, L. Sun, Y. Zhang, T. Li, J. Kim, C.-Y. Chim, G. Galli, F. Wang, Emerging photoluminescence in monolayer MoS, Nano Lett. 10 (2010) 1271-1275.

[6] W.J. Zhao, Z. Ghorannevis, L.Q. Chu, M.L. Toh, C. Kloc, P.H. Tan, G. Eda, Evolution of electronic structure in atomically thin sheets of $\mathrm{WS}_{2}$ and $\mathrm{WSe}_{2}, \mathrm{ACS}$ Nano 7 (2013) 791-797.

[7] A. Ramasubramaniam, Large excitonic effects in monolayers of molybdenum and tungsten dichalcogenides, Phys. Rev. B 86 (2012)

[8] A. Kuc, N. Zibouche, T. Heine, Influence of quantum confinement on the electronic structure of the transition metal sulfide $\mathrm{TS}_{2}$, Phys. Rev. B 83 (2011).

[9] K.K. Kam, B.A. Parkinson, Detailed photocurrent spectroscopy of the semiconducting Group-Vi transition-metal dichalcogenides, J. Phys. Chem.: US 86 (1982) 463-467

[10] S.-W. Min, H.S. Lee, H.J. Choi, M.K. Park, T. Nam, H. Kim, S. Ryu, S. Im, Nanosheet thickness-modulated $\mathrm{MoS}_{2}$ dielectric property evidenced by field-effect transistor performance, Nanoscale 5 (2013) 548

[11] H. Li, Z. Yin, Q. He, H. Li, X. Huang, G. Lu, D.W.H. Fam, A.I.Y. Tok, Q. Zhang, H. Zhang, Layered nanomaterials fabrication of single- and multilayer MoS film-based field-effect transistors for sensing NO at room temperature, Small 8 (2012) 63-67

[12] H. Liu, J.J. Gu, P.D. Ye, $\mathrm{MoS}_{2}$ nanoribbon transistors: transition from depletion mode to enhancement mode by channel-width trimming, IEEE Electron Dev. Lett. 33 (2012) 1273-1275.

[13] G. Eda, H. Yamaguchi, D. Voiry, T. Fujita, M. Chen, M. Chhowalla, Photoluminescence from chemically exfoliated MoS2, Nano Lett. 11 (2011) 5111-5116.

[14] R.S. Sundaram, M. Engel, A. Lombardo, R. Krupke, A.C. Ferrari, P. Avouris, M. Steiner, Electroluminescence in single layer $\mathrm{MoS}_{2}$, Nano Lett. 13 (2013) 1416-1421.

[15] J.N. Coleman, M. Lotya, A. O’Neill, S.D. Bergin, P.J. King, U. Khan, K. Young, A. Gaucher, S. De, R.J. Smith, I.V. Shvets, S.K. Arora, G. Stanton, H.-Y. Kim, K. Lee, G.T Kim, G.S. Duesberg, T. Hallam, J.J. Boland, J.J. Wang, J.F. Donegan, J.C. Grunlan, G. Moriarty, A. Shmeliov, R.J. Nicholls, J.M. Perkins, E.M. Grieveson, K. Theuwissen, D.W. McComb, P.D. Nellist, V. Nicolosi, Two-dimensional nanosheets produced by liquid exfoliation of layered materials, Science 331 (2011) 568-571.

[16] R.J. Smith, P.J. King, M. Lotya, C. Wirtz, U. Khan, S. De, A. O’Neill, G.S. Duesberg, J.C. Grunlan, G. Moriarty, J. Chen, J. Wang, A.I. Minett, V. Nicolosi, J.N. Coleman, Large-scale exfoliation of inorganic layered compounds in aqueous surfactant solutions, Adv. Mater. 23 (2011) 3944-3948.

[17] Z.Zeng, T. Sun, J. Zhu, X. Huang, Z. Yin, G. Lu, Z. Fan, Q. Yan, H.H. Hng, H. Zhang, An effective method for the fabrication of few-layer-thick inorganic nanosheets, Angew. Chem. Int. Ed. 51 (2012) 9052-9056.

[18] Z. Zeng, Z. Yin, X. Huang, H. Li, Q. He, G. Lu, F. Boey, H. Zhang, Single-layer semiconducting nanosheets: high-yield preparation and device fabrication, Angew. Chem. Int. Ed. 50 (2011) 11093-11097.

[19] T. Drescher, F. Niefind, W. Bensch, W. Grünert, Sulfide catalysis without coordinatively unsaturated sites: hydrogenation, cis-trans isomerization, and $\mathrm{H}_{2} / \mathrm{D}_{2}$ scrambling over $\mathrm{MoS}_{2}$ and WS 2 , J. Am. Chem. Soc. 134 (2012) 18896-18899.

[20] G. Cunningham, M. Lotya, N. McEvoy, G.S. Duesberg, P. van der Schoot, J.N. Coleman, Percolation scaling in composites of exfoliated $\mathrm{MoS}_{2}$ filled with nanotubes and graphene, Nanoscale 4 (2012) 6260

[21] X. Huang, Z.Y. Zeng, S.Y. Bao, M.F. Wang, X.Y. Qi, Z.X. Fan, H. Zhang, Solutionphase epitaxial growth of noble metal nanostructures on dispersible singlelayer molybdenum disulfide nanosheets, Nat. Commun. 4 (2013) (article no. 1444).

[22] Z. Zeng, C. Tan, X. Huang, S. Bao, H. Zhang, Growth of noble metal nanoparticles on single-layer $\mathrm{TiS}_{2}$ and $\mathrm{TaS}_{2}$ nanosheets for hydrogen evolution reaction, Energy Environ. Sci. (2014), http://dx.doi.org/10.1039/C1033EE42620C (advance article)

[23] W.J. Zhou, Z. Y Yin, Y.P. Du, X Huang, ZY Zeng, ZX. Fan, H. Liu, J. . Wang $\mathrm{H}$. Zhang, Synthesis of few-layer $\mathrm{MoS}_{2}$ nanosheet-coated $\mathrm{TiO}_{2}$ nanobelt heterostructures for enhanced photocatalytic activities, Small 9 (2013) 140-147.

[24] K. Chang, W. Chen, Single-layer $\mathrm{MoS}_{2}$ /graphene dispersed in amorphous carbon: towards high electrochemical performances in rechargeable lithium ion batteries, J. Mater. Chem. 21 (2011) 17175-17184.

[25] X. Cao, Y. Shi, W. Shi, X. Rui, Q. Yan, J. Kong, H. Zhang, Preparation of $\mathrm{MoS}_{2}$-coated three-dimensional graphene networks for high-performance anode material in lithium-ion batteries, Small 9 (2013) 3433-3438.

[26] L. Cao, S. Yang, W. Gao, Z. Liu, Y. Gong, L. Ma, G. Shi, S. Lei, Y. Zhang, S. Zhang, R. Vajtai, P.M. Ajayan, Direct laser-patterned micro-supercapacitors from paintable $\mathrm{MoS}_{2}$ films, Small(2013), http://dx.doi.org/10.1002/smll.201203164.

[27] S. Kumar, N. Peltekis, K. Lee, H.-Y. Kim, G. Duesberg, Reliable processing of graphene using metal etchmasks, Nanoscale Res. Lett. 6 (2011) 390.

[28] S. Kumar, N. McEvoy, T. Lutz, G.P. Keeley, V. Nicolosi, C.P. Murray, W.J. Blau, G.S. Duesberg, Gas phase controlled deposition of high quality large-area graphene films, Chem. Commun. 46 (2010) 1422-1424

[29] X. Li, W. Cai, J. An, S. Kim, J. Nah, D. Yang, R. Piner, A. Velamakanni, I. Jung, E. Tutuc, S.K. Banerjee, L. Colombo, R.S. Ruoff, Large-area synthesis of high-quality and uniform graphene films on copper foils, Science 324 (2009) 1312-1314. 
G Model

APSUSC-27111; No. of Pages 8

8

R. Gatensby et al. / Applied Surface Science $x x x(2014) x x x-x x x$

[30] D. Kong, H. Wang, J.J. Cha, M. Pasta, K.J. Koski, J. Yao, Y. Cui, Synthesis of $\mathrm{MoS}_{2}$ and $\mathrm{MoSe}_{2}$ films with vertically aligned layers, Nano Lett. 13 (2013) 1341-1347.

[31] Y. Zhan, Z. Liu, S. Najmaei, P.M. Ajayan, J. Lou, Large-area vapor-phase growth and characterization of $\mathrm{MoS}_{2}$ atomic layers on a $\mathrm{SiO}_{2}$ substrate, Small 8 (2012) 966-971.

[32] M.R. Laskar, L. Ma, S. Kannappan, P. Sung Park, S. Krishnamoorthy, D.N. Nath, W. Lu, Y. Wu, S. Rajan, Large area single crystal (0001) oriented $\mathrm{MoS}_{2}$, Appl. Phys. Lett. 102 (2013).

[33] Y.-H. Lee, L. Yu, H. Wang, W. Fang, X. Ling, Y. Shi, C.-T. Lin, J.-K. Huang, M.-T. Chang, C.-S. Chang, M. Dresselhaus, T. Palacios, L.-J. Li, J. Kong, Synthesis and transfer of single-layer transition metal disulfides on diverse surfaces, Nano Lett. (2013) 130329103804007.

[34] Y.-H. Lee, X.-Q. Zhang, W. Zhang, M.-T. Chang, C.-T. Lin, K.-D. Chang, Y.-C. Yu, J.T.-W. Wang, C.-S. Chang, L.-J. Li, T.-W. Lin, Synthesis of large-area $\mathrm{MoS}_{2}$ atomic layers with chemical vapor deposition, Adv. Mater. 24 (2012) 2320-2325.

[35] J. Mann, D. Sun, Q. Ma, J.-R. Chen, E. Preciado, T. Ohta, B. Diaconescu, K. Yamaguchi, T. Tran, M. Wurch, K. Magnone, T.F. Heinz, G.L. Kellogg, R. Kawakami, L. Bartels, Facile growth of monolayer $\mathrm{MoS}_{2}$ film areas on $\mathrm{SiO}_{2}$, Eur. Phys. J. B 86 (2013) 1-4.

[36] S. Najmaei, Z. Liu, W. Zhou, X. Zou, G. Shi, S. Lei, B.I. Yakobson, J.C. Idrobo, P.M. Ajayan, J. Lou, Vapour phase growth and grain boundary structure of molybdenum disulphide atomic layers, Nat. Mater. 12 (2013) 754-759.

[37] W.Y. Lee, T.M. Besmann, M.W. Stott, Preparation of $\mathrm{MoS}_{2}$ thin-films by chemical vapor deposition, J. Mater. Res. 9 (1994) 1474-1483.

[38] K.-K. Liu, W. Zhang, Y.-H. Lee, Y.-C. Lin, M.-T. Chang, C.-Y. Su, C.-S. Chang, H. Li, Y. Shi, H. Zhang, C.-S. Lai, L.-J. Li, Growth of large-area and highly crystalline $\mathrm{MoS}_{2}$ thin layers on insulating substrates, Nano Lett. 12 (2012) 1538-1544.

[39] M. Shanmugam, T. Bansal, C.A. Durcan, B. Yu, Schottky-barrier solar cell based on layered semiconductor tungsten disulfide nanofilm, Appl. Phys. Lett. 101 (2012) 263902-263905

[40] T.W. Scharf, S.V. Prasad, M.T. Dugger, P.G. Kotula, R.S. Goeke, R.K. Grubbs, Growth, structure, and tribological behavior of atomic layer-deposited tungsten disulphide solid lubricant coatings with applications to MEMS, Acta Mater. 54 (2006) 4731-4743.

[41] J. Kong, N.R. Franklin, C.W. Zhou, M.G. Chapline, S. Peng, K.J. Cho, H.J. Dai, Nanotube molecular wires as chemical sensors, Science 287 (2000) 622-625.

[42] H. Li, Z.Y. Yin, Q.Y. He, X. Huang, G. Lu, D.W.H. Fam, A.I.Y. Tok, Q. Zhang, H. Zhang, Fabrication of single- and multilayer $\mathrm{MoS}_{2}$ film-based field-effect transistors for sensing NO at room temperature, Small 8 (2012) 63-67.
[43] F. Schedin, A.K. Geim, S.V. Morozov, E.W. Hill, P. Blake, M.I. Katsnelson, K.S. Novoselov, Detection of individual gas molecules adsorbed on graphene, Nat. Mater. 6 (2007) 652-655.

[44] D.R. Kauffman, A. Star, Carbon nanotube gas and vapor sensors, Angew. Chem. Int. Ed. 47 (2008) 6550-6570.

[45] K.R. Ratinac, W.R. Yang, S.P. Ringer, F. Braet, Toward ubiquitous environmental gas sensors-capitalizing on the promise of graphene, Environ. Sci. Technol. 44 (2010) 1167-1176.

[46] T. Zhang, S. Mubeen, N.V. Myung, M.A. Deshusses, Recent progress in carbon nanotube-based gas sensors, Nanotechnology 19 (2008).

[47] F.K. Perkins, A.L. Friedman, E. Cobas, P.M. Campbell, G.G. Jernigan, B.T. Jonker, Chemical vapor sensing with monolayer $\mathrm{MoS}_{2}$, Nano Lett. 13 (2013) 668-673.

[48] D.J. Late, Y.-K. Huang, B. Liu, J. Acharya, S.N. Shirodkar, J. Luo, A. Yan, D. Charles, U.V. Waghmare, V.P. Dravid, C.N.R. Rao, Sensing behavior of atomically thinlayered $\mathrm{MoS}_{2}$ transistors, ACS Nano 7 (2013) 4879-4891.

[49] Q. He, Z. Zeng, Z. Yin, H. Li, S. Wu, X. Huang, H. Zhang, Fabrication of flexible $\mathrm{MoS}_{2}$ thin-film transistor arrays for practical gas-sensing applications, Small 8 (2012) 2994-2999.

[50] Y. Yao, Z. Lin, Z. Li, X. Song, K.-S. Moon, C.-p. Wong, Large-scale production of two-dimensional nanosheets, J. Mater. Chem. 22 (2012) 13494-13499.

[51] K. Lee, R. Gatensby, N. McEvoy, T. Hallam, G.S. Duesberg, High performance sensors based on molybdenum disulfide thin films, Adv. Mater. 25 (2013) 6699-6702.

[52] M.M. Mdleleni, T. Hyeon, K.S. Suslick, Sonochemical synthesis of nanostructured molybdenum sulfide, J. Am. Chem. Soc. 120 (1998) 6189-6190.

[53] H. Li, Q. Zhang, C.C.R. Yap, B.K. Tay, T.H.T. Edwin, A. Olivier, D. Baillargeat, From bulk to monolayer $\mathrm{MoS}_{2}$ : evolution of raman scattering, Adv. Funct. Mater. 22 (2012) 1385-1390.

[54] A. Molina-Sánchez, L. Wirtz, Phonons in single-layer and few-layer $\mathrm{MoS}_{2}$ and $W_{2}$, Phys. Rev. B 84 (2011) 155413.

[55] M.A. Baker, R. Gilmore, C. Lenardi, W. Gissler, XPS investigation of preferential sputtering of S from $\mathrm{MoS}_{2}$ and determination of $\mathrm{MoS}_{X}$ stoichiometry from Mo and S peak positions, Appl. Surf. Sci. 150 (1999) 255-262.

[56] J.W. Chung, Z.R. Dai, F.S. Ohuchi, WS2 thin films by metal organic chemical vapor deposition, J. Cryst. Growth 186 (1998) 137-150.

[57] J. Li, Y.J. Lu, Q. Ye, M. Cinke, J. Han, M. Meyyappan, Carbon nanotube sensors for gas and organic vapor detection, Nano Lett. 3 (2003) 929-933.

[58] L.A. Currie, Nomenclature in evaluation of analytical methods including detection and quantification capabilities (IUPAC Recommendations 1995), Pure Appl. Chem. 67 (1995) 1699-1723.

Please cite this article in press as: R. Gatensby, et al., Controlled synthesis of transition metal dichalcogenide thin films for electronic applications, Appl. Surf. Sci. (2014), http://dx.doi.org/10.1016/j.apsusc.2014.01.103 